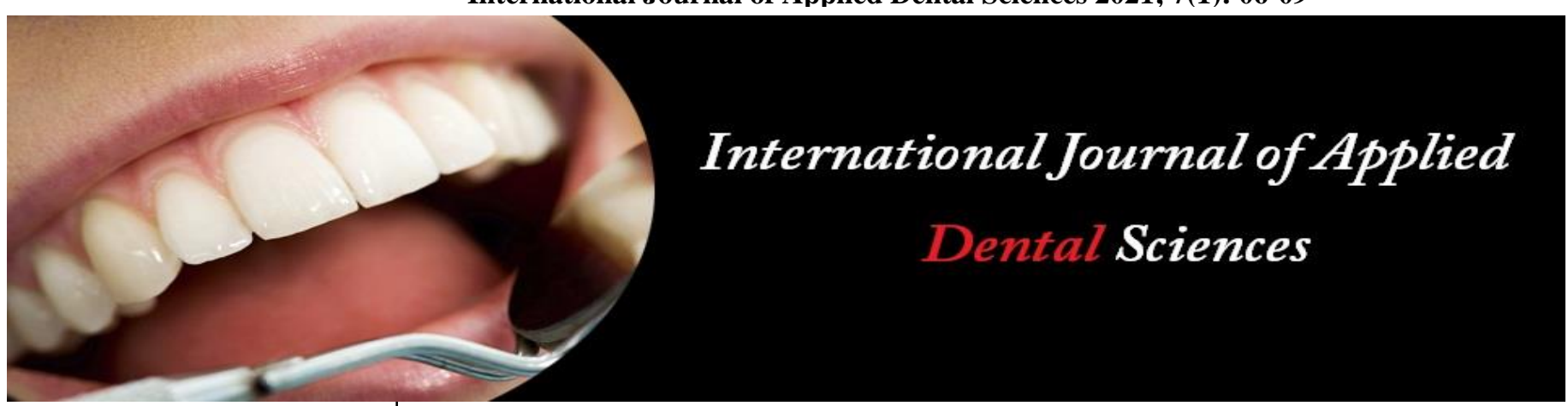

ISSN Print: 2394-7489

ISSN Online: 2394-7497

IJADS 2021; 7(1): 06-09

(C) 2021 IJADS

www.oraljournal.com

Received: 04-11-2020

Accepted: 06-12-2020

Dr. Siddharth Kumar Singh

Professor, Department of Oral

Medicine and Radiology,

Saraswati Dental College,

Lucknow, Uttar Pradesh, India

Dr. Sunira Chandra

Professor and Head, Department

of Oral Medicine and Radiology,

Saraswati Dental College,

Lucknow, Uttar Pradesh, India

Dr. Vaishali Kesarwani

Intern, Saraswati Dental College,

Lucknow, Uttar Pradesh, India

Dr. Preeti Agrahari

Intern, Saraswati Dental College, Lucknow, Uttar Pradesh, India

Corresponding Author: Dr. Siddharth Kumar Singh Professor, Department of Oral Medicine and Radiology, Saraswati Dental College, Lucknow, Uttar Pradesh, India

\section{Novel coronavirus (COVID-19) and dentistry}

\author{
Dr. Siddharth Kumar Singh, Dr. Sunira Chandra, Dr. Vaishali Kesarwani \\ and Dr. Preeti Agrahari
}

DOI: $\underline{\text { https://doi.org/10.22271/oral.2021.v7.i1a.1108 }}$

\section{Abstract}

SARS-CoV-2 is a member of the family of coronaviruses. The first cases were recorded in Wuhan, China, between December 2019 and January 2020. COVID-19 is a new challenge in modern dentistry. New guidelines are required in dental clinics to avoid contagion caused by cross-infections. Precautionary measures should be applied to control COVID-19 in clinical practice. Several authors have highlighted the importance of tele- triage and/or clinic questionnaires, body temperature measurement, usage of personal protective equipment, surface disinfection with ethanol between $62 \%$ and $71 \%$, highspeed instruments equipped with an anti-retraction system, four-handed work, and large-volume cannulas for aspiration. Clinically, the use of a rubber dam is essential. FFP2 (or N95) and FFP3 respirators, if compared to surgical masks, provide greater protection for health workers against viral respiratory infections. Further studies are needed to confirm this.

Keywords: Covid-19, infection, PPE, dental practice

\section{Introduction}

By the end of 2019, a pneumonia outbreak with unknown etiology occurred in Wuhan, China. Most of the cases were linked to a local seafood market selling live animals, suggesting that the pathogens were transmitted from animals to humans, soon escalating to human-to-human transmission. The pathogen was identified and named as 2019 novel coronavirus (2019$\mathrm{nCoV}$ ), and the disease was named corona virus disease 2019 (COVID-19), which stands for coronavirus disease 2019. As of now, December 2020, there were 10.1 million confirmed cases of COVID-19 in India. (WHO, 2020) As the number of confirmed cases continues to rise, global health, economic development, and even social stability are falling under immense pressure. On January 31, 2020, the WHO declared the COVID-19 crisis as a "Public Health Emergency of International Concern." As stated in the 6th Edition of COVID-19 Treatment Regimen (Trial Implementation) published by the National Health Commission of the People's Republic of China (2020), the possible routes of 2019- nCoV transmission are mainly direct contact and droplet transmission. Aerosol transmission is also a possible route of transmission when there is an exposure to high concentrations of aerosols in a relatively closed environment [1]. Routine dental procedures generate aerosols, which pose potential risks to the dental care personnel and patients. Although there are no reported cases of coronavirus transmission in a dental setting, given the high transmissibility of the disease, dental teams should be alert and maintain a healthy environment for both the patients and themselves. Therefore, understanding aerosol transmission and its implications in dentistry is essential. In addition to standard precautions, some special precautions should also be implemented during this special period [2].

\section{Potential routes of transmission}

Aerosol transmission and its implication in dentistry

Droplets and aerosols play a significant role in the transmission of diseases. When a person coughs, sneezes, laughs, or talks, large ( $>5 \mu \mathrm{m}$ diameter) and small $(\leq 5 \mu \mathrm{m}$ diameter) droplets or aerosols are generated. Due to gravity, larger droplets fall to the ground quickly; therefore, droplet transmission requires close physical proximity between an infected individual and a 
susceptible individual. On the other hand, small droplets or small particle residues of evaporated droplets have a low settling velocity, so they may remain in the air for a longer time and travel further before they can enter the respiratory tract or contaminate surfaces (WHO, 2014). Results from some studies have shown that aerosols from highly virulent pathogens like severe acute respiratory syndrome-coronavirus (SARSCoV) can travel more than six feet contaminated surfaces have been found to be a route of transmission of several nosocomial pathogens. Although human coronaviruses including SARS-CoV and Middle East respiratory syndrome-coronavirus (MERS-CoV) have limited capacity to survive on a dry surface, several studies have reported that they can persist on a surface for a few days, particularly when suspended in human secretion, and undergo onward transmission. Hand contact with contaminated surfaces may lead to pathogen acquisition and transfer to the eyes, nose, or mouth, resulting in a new case of infection ${ }^{[2,3]}$.

\section{Droplets and aerosols in dental setting}

When performing dental procedures with a high speed hand piece, friction between the tooth and the rapidly rotating bur would create excessive heat. Without a coolant, the heat could cause damage to hard dental tissue and lead to pathological changes to the dental pulp. Therefore, to prevent heat gain, it is a universal consensus to use a water coolant when performing dental procedures, including tooth preparation, oral prophylaxis, and oral surgery ${ }^{[4]}$. The water coolant, however, could generate aerosols. When combined with bodily fluids in the oral cavity, such as blood and saliva, bio aerosols are created These bio aerosols are commonly contaminated with bacteria, fungi, and viruses, and have the potential to float in the air for a considerable amount of time and be inhaled by the dentists or other patients ${ }^{[5]}$. A review conducted by showed that 38 types of microorganisms could be found in the air of the dental clinic, including Legionella pneumophila, the causative agent of severe pneumonia. There have been reports of patients acquiring pneumonia after being treated at a dental clinic ${ }^{[6]}$. Past evidence showed that the majority of SARS-CoV and MERS-CoV cases were associated with nosocomial transmission in hospitals, resulting, partly, from the use of aerosol-generating procedures performed on patients with respiratory disease ${ }^{[7]}$. Based on the current epidemiological data, 2019-nCoV has higher transmissibility than SARS-CoV and MERS-CoV ${ }^{[8]}$. Therefore, modification of standard precaution and infection control regimen targeted toward 2019- $\mathrm{nCoV}$ is essential during this outbreak ${ }^{[1]}$.

\section{Immediate impact of COVID-19 on dental practice}

Dental practitioners are exposed to high risks of contracting COVID-19, due to their direct exposure to saliva and blood. At the moment, several countries have issued some levels of quarantine measures, to "flatten the curve" during this first outbreak, and dentists have been instructed to only provide emergency treatments for the time being. Going forward, as SARS-CoV-2 is going to represent a menace presumably until a vaccine is developed and distributed, dental practitioners have to adapt their practices to protect themselves and their patients from this infection. This minireview searched the literature for procedures that could significantly reduce the risk of an operator or a dental assistant to get contaminated, while at the same time protecting their patients ${ }^{[9]}$. These dental emergency categories included:

- Severe and uncontrolled pain;
- Spreading, recurrent or continuing infection;

- Avulsed permanent tooth;

- Severe trauma.

It was also advised that all community outreach activities such as oral health improvement programs should be stopped until advised otherwise. UK General Dental Council (GDC) released some guidelines for remote consultation and prescription. It was suggested that patient safety must be the priority, and the identity of each patient must be checked and verified. Dentists should be able to collect sufficient information regarding patient's health and conditions in order to be able to prescribe the medication safely. It is also important to identify vulnerable patients and take appropriate steps to protect them including obtaining valid and informed consent and following relevant mental capacity law and codes of practice by all prescribing professionals. This guideline also provided further details regarding the information that is required to be provided to the patients for each e-Consultation and prescription, including note keeping that must be clear and should justify the clinician's decisions. One of the limitations of the remote service was that the General Pharmaceutical Council produced a list of medicines that could not be prescribed remotely unless some safeguarding measures were implemented. This document explained that pharmacies based in England, Scotland and Wales may not supply these categories of medicines without having an assurance that these safeguards are in place ${ }^{[9]}$.

\section{PPE for treating COVID-19 patients}

SARS-CoV-2 infection may occur through direct or indirect contact and aerosol, therefore when the outbreak is at community level Transmission-Based Precautions for contact, droplets and airborne must be taken for all patients. Standard surgical masks are effective against splashes and large-sized droplets but not effective against small airborne particles thus fit-tested N-95 respirators or superior ones are required; even more when aerosol-producing treatments are performed. Protective disposable impermeable gowns and caps are recommended as well as gloves and eye protection. These medical devices should be worn before coming in contact with the patient and discarded safely before leaving the room. It is paramount that all the staff is adequately instructed on the use and doffing of Personal Protective Equipment (PPE) as deviation from standard procedures will increase the risk of infection ${ }^{[10]}$.

*Potential role of dentists and patients in reducing the spread of COVID-19

\section{Strict protocol for patient Screening}

Dental professionals must be able to screen and identify potential high-risk COVID-19 patients to prevent the spread of the infectious disease. No routine dental treatment should be carried out on patients at the early stages of infection, and these patients should be encouraged to quarantine and selfisolate themselves ${ }^{[9]}$. The following questions should be asked:

1. Have you experienced fever or symptoms like cough, shortness of breath or other respiratory problems in the past 14 days?

2. Have you been in contact with someone affected by COVID-19 in the past 14 days?

3. Have you been in contact with someone reporting fever or respiratory problems in the past 14 days?

4. Did you participate in gatherings, meetings or had contact with many unacquainted people in the past 14 days? 
5. Did you travel from areas with Level 3 Travel Health Notice for COVID-19? (This question has been omitted when the virus is declared at community level).

If the patient answers yes to any of these questions the treatment should be postponed and the patient instructed to contact his physician if the answer to questions number one or two is positive ${ }^{[10]}$.

\section{Hand hygiene}

Indirect transmission by touching infected surfaces is one of the proven methods of transmission of airborne diseases therefore, reinforced hand hygiene must be made compulsory and all members of the dental team and other staff should follow the guidelines in place ${ }^{[11]}$.

Hand hygiene is a critical measure for reducing SARS-CoV-2 transmission. It is crucial to perform thorough hand washing when coming into contact with patients and no disinfected surfaces or equipment, and it is recommended to avoid touching eyes, mouth, and nose without having hands carefully washed. In particular, a protocol involving 5 hand washings was proposed to reinforce professional's compliance ${ }^{[12]}$.

\section{Mouth rinse before dental procedures}

Use of mouthwash prior to treatment reduces the number of microorganisms in the mouth and subsequently decreases airborne microorganisms following dental treatments. Chlorhexidine is commonly suggested as a pre-treatment mouthwash. Oxidizing mouthwashes such as hydrogen peroxide $1 \%$, povidone or cetylpyridinium chloride (CPC) $0.10 \%$ to $0.05 \%$ are recommended for protection against Corona viruses. Povidone-iodine can safely be used in the mouth with up to $2.5 \%$ and it rapidly inactivates coronaviruses when applied for 15 seconds ${ }^{[11]}$.

\section{Rubber dam isolation}

During dental procedures that generate aerosols, rubber dam provides barrier protection from the primary source and will virtually eliminate all pathogens emerging from respiratory secretion. If the rubber dam is placed correctly, the only source of contamination would be the tooth that is undergoing treatment ${ }^{[13]}$. Application of rubber dam during cavity preparation showed a significant reduction in the spread of microorganisms by $90 \%{ }^{[2]}$. Rubber dam is applied in all aerosol-generating procedures. One disadvantage of using the rubber dam is that it is not feasible in procedures that require sub gingival instrumentation, such as sub gingival restoration and sub gingival crown margin preparation ${ }^{[1]}$.

\section{Anti-retraction hand piece}

Peng et al. highlighted the risk related to the performance of dental procedures, in particular when hand pieces and ultrasonic devices are employed. As reported by Meng et al., it is advisable to minimize the operations involving the generation of aerosol and droplets while employing use of personal protective equipment ${ }^{[14]}$. In general, the use of antiretraction hand pieces is recommended for dental treatments for waterline protocols ${ }^{[11]}$.

\section{Strict disinfecting protocol for clinical environment}

During aerosol generating procedures, droplets containing infective pathogens could be deposited on the surrounding surfaces. An analysis of 22 studies revealed that human coronaviruses, such as SARS and MERS, can persist on inanimate surfaces for up to $9 \mathrm{~d}$. However, they can be efficiently inactivated by surface disinfects within one minute. These surface disinfectants contain $62 \%-71 \%$ ethanol, $0.5 \%$ hydrogen peroxide, and $0.1 \%(1 \mathrm{~g} / \mathrm{L})$ sodium. Surfaces are disinfected after each patient visits, especially surfaces in close proximity to the operating areas ${ }^{[2,15]}$.

\section{Clinical waste management}

Medical waste is divided into two categories: infectious and non-infectious. The existing guidelines seem to be sufficient and must be closely executed. Clinical waste should be stored in a safe temporary storage area, and all reusable instruments and items should be pre-treated, cleaned, sterilized and properly stored in accordance with the local protocols. The clinical waste generated after treatment of COVID-19 positive patients must be regarded as infectious clinical waste and stored in clinical waste bags within a designated area. The surface of the package bags should be marked and disposed according to the local regulations and requirement for the management of medical waste ${ }^{[11,16]}$.

\section{Conclusion}

COVID-19 is a major concern for public health and dentists are among the healthcare professionals facing the greatest risks of infection. Dentists, by nature, are at high risk of exposure to infectious diseases. The emergence of COVID-19 has brought new challenges and responsibilities to dental professionals.

\section{References}

1. $\mathrm{Ng}$ MY, Lee EY, Yang J. Imaging profile of the COVID19 infection: radiologic findings and literature review. Radiol Cardiothorac Imaging 2020;2(1):20-34.

2. Zi-yu GE. Possible aerosol transmission of COVID-19 and special precautions in dentistry. J Zhejiang Univ-Sci B (Biomed \& Biotechnol) 2020;21(5):361-368.

3. Otter JA, Yezli S, Salkeld JAG. Evidence that contaminated surfaces contribute to the transmission of hospital pathogens and an overview of strategies to address contaminated surfaces in hospital settings. Am J Infect Control 2013;41(5):6-11.

4. Farah RI. Effect of cooling water temperature on the temperature changes in pulp chamber and at hand piece head during high-speed tooth preparation. Restor Dent Endod 2019,44(1).

5. Grenier D. Quantitative analysis of bacterial aerosols in two different dental clinic environments. Appl Environ Microbiol 1995;61(8):3165-3168.

6. Zemouri C, de Soet H, Crielaard W. A scoping review on bio-aerosols in healthcare and the dental environment. PLoS one 2017;12(5):78-88.

7. Chowell G, Abdirizak F, Lee S. Transmission characteristics of MERS and SARS in the healthcare setting: a comparative study. BMC Med 2015;13:210-15.

8. Chen JL. Pathogenicity and transmissibility of 2019nCoV-a quick overview and comparison with other emerging viruses. Microbes Infect 2020;8:1-18.

9. Barabari P, Moharamzadeh K. Novel Coronavirus (COVID-19) and Dentistry-A Comprehensive Review of Literature. Dent. J 2020;8(53):1-18.

10. Careddu R, Ciaschetti M, Creavin G, Molina F, Plotino G. COVID-19 and dental practice: overview and protocols during pandemic. Giornale Italiano di Endodonzia 2020;34:1-6.

11. Ashtiani RE, Tehrani S, León MR, Zandinejad A. 
Reducing the Risk of COVID-19 Transmission in Dental Offices: A Review. Journal of Prosthodontics 2020,1-7.

12. Peng $\mathrm{X}, \mathrm{Xu} \mathrm{X}, \mathrm{Li} \mathrm{Y}$, Cheng L, Zhou $\mathrm{X}$, Ren $\mathrm{B}$. Transmission routes of 2019-nCoV and controls in dental practice. Int J Oral Sci 2020;12(1):9.

13. Harrel SK, Molinari J. Aerosols and splatter in dentistry: a brief review of the literature and infection control implications. J Am Dent Assoc 2004;135(4):429-437.

14. Izzetti R, Nisi M, Gabriele M, Graziani F. COVID-19 Transmission in Dental Practice: Brief Review of Preventive Measures in Italy. Journal of Dental Research 2020;99(9):1030-1038

15. Passarelli PC, Rella E, Manicone PF, Godoy PG, Addona AD. The impact of the COVID-19 infection in dentistry. Experimental Biology and Medicine 2020;245:940-944.

16. Villani FA, Aiuto R, Paglia L, Re D. COVID-19 and Dentistry: Prevention in Dental Practice, a Literature Review. Int. J. Environ. Res. Public Health 2020;17:1-12. 